\title{
Role of Robotic Surgery in Gynecologic Oncology in India
}

\author{
R Anjali
}

\begin{abstract}
Aim: This article aimed to study the role of robotic surgery in gynecologic oncology in India over the past decade.

Background: Different randomized and observational, retrospective and prospective studies that met the eligibility criteria were included. Various parameters were compared between robotic and laparoscopic surgeries. The different parameters evaluated in the studies were operative time, estimated blood loss, hospital stay, complications, conversion rates, so on and so forth. Nodal yield, vaginal margin and paracervical clearance were studied in a few of them. PubMed was the main search engine utilized for searching the study data.

Review results: After careful analysis of the data, it was noted that the complication rate, blood loss, and postsurgery hospitalization were significantly lower with robotics, whereas some inconsistencies were noted regarding the operating time.

Conclusion: India is notably at the brink of a revolution. The need of the hour is to make this new surgically innovative technology accessible to all-to the surgeons as well as the patients.

Clinical significance: Critical analysis of robotic surgeries in gynecology in Indian setting has been done. This would help in planning adoption and training of this upcoming domain.

Keywords: Da vinci robotic surgical system, Gynecologic oncology, Robotic surgery.

World Journal of Laparoscopic Surgery (2018): 10.5005/jp-journals-10033-1345
\end{abstract}

\section{INTRODUCTION}

In recent times, through robotic surgery, medical quality of care has taken a giant leap towards the better though limited at present by cost factor. Robotic surgery has superseded laparoscopic surgeries due to various reasons such as 3D vision, tremor filtering, precise movements of the instruments with seven degrees of movement and many others. The state-of-art da Vinci robotic surgical system (DRS) heralds the beginning of a new era which could possibly mean the end of laparoscopic and open surgeries as we know them.

There are innumerable robots in the USA alone with an exponential rise in their utilization rates. Their popularity has also spread to Europe, Asia and Australia. Currently in India, robotic surgery is in the early developmental and adaptive phase. As per the data for this year, there are 19 robots in India-in New Delhi, Gurugram, Mumbai, Chennai, Nadiad, Bengaluru, and Hyderabad. The All India Institute of Medical Sciences, New Delhi, has contributed a lot to the progress in robotic surgery in our country. It was here that the first robotic prostatectomy in India was performed in July 2006. ${ }^{1}$ From then on, the trend is catching on but financial constraint is the main limitation. The future prospects with new upcoming robots do sound promising.

\section{Robotic SURgery vs Laparoscopic APPROACH}

The major contributing factor to the unprecedented explosion in the use of robotic surgery is its unquestionable superiority over conventional laparoscopic surgery. It basically overcomes most of the limitations of laparoscopic surgery. It offers many advantages to the surgeon as well as the patient. Unlike laparoscopy (which has a disheartening learning curve), open surgeons can easily switch over to robotics due to its various advantages over other MAS (minimal access surgery). It offers a high degree of magnification; has a surgeon-friendly profile with better ergonomics; and has EndoWrist technology, which provides a whole range of movements
Department of Obstetrics and Gynecology, Sapthagiri Institute of Medical Sciences and Research Institute, Bengaluru, Karnataka, India

Corresponding Author: R Anjali, Department of Obstetrics and Gynecology, Sapthagiri Institute of Medical Sciences and Research Institute, Bengaluru, Karnataka, India, Phone: +91 8431974145, e-mail: dr.anjaliramaswamy@gmail.com

How to cite this article: Anjali R. Role of Robotic Surgery in Gynecologic Oncology in India. World J Lap Surg 2018;11(3):135-137.

Source of support: Nil

Conflict of interest: None

with seven degrees of freedom; so on and so forth. ${ }^{2}$ Owing to the aforementioned reasons, this master-slave surgical robot has widespread applications in all surgical fields, especially where intracorporeal suturing is required as it provides unmatched meticulous anatomy of the surgical field of interest. When a complex condition is encountered in clinical practice, it is our general tendency to avoid MAS and proceed with open surgeries. This is where the robots step in and prove as a very valuable tool for a better outcome with minimal chances of complication.

From a patient's point of view, owing to the higher cost one may feel that it is indeed a marketing strategy. But there are many evidence-based studies that establish advantages to the patient without any element of doubt. DRS needs smaller incisions, has lesser blood loss, shorter hospital stay and is associated with less pain. $^{3}$

\section{Initiation of Robotic Surgery}

The DRS was brought into the market by Intuitive Surgical Systems, Inc., and was US FDA cleared for urologic procedures in 2001 and gynecologic surgery in $2005 .{ }^{4}$ Urologists were the first among the medical fraternity to accept this technology whole heartedly; prostatectomy being the first surgery performed by them. Incidentally it still remains the most common procedure 
being performed with DRS. Department of Gynecology is not lagging behind with scores of surgeries being performed with robotic assistance, to mention among those are the gynecologic oncology surgeries. The utility of DRS is more so in urology and gynecology as robotics is best for single quadrant surgery and for fixed structures. Other surgical fields are also making promising progress with the total number of robotic surgeries on the rise worldwide.

\section{Robotic Surgery in Gynecology}

In gynecology, hysterectomy remains the hallmark surgery. Though noteworthy advances are being made in general gynecology, reproductive gynecology, and reconstructive gynecology, much of the focus still remains on gynecologic oncology and the role of DRS in gynecology is still expanding.

It was noted that in hysterectomies done for benign conditions, with robotics there were significantly lesser operative times, blood loss and conversion rates. Since robotics gives better results with intracorporeal suturing, it is especially useful in tubal anasthamosis that requires precision and extensive suturing. But further studies are needed to validate its use and consequent pregnancy outcomes. In myomectomy, robotic surgery offers many advantages compared with traditional laparoscopy in the form of better enucleation due to better dexterity, better intracorporeal suturing, less blood loss, and hospital stay. DRS is expected to play a significant role in the most recent upcoming subdivision of Obstetrics and Gynecology-Pelvic Reconstructive Surgery. Particularly procedures such as sacrocolpopexy and vesicovaginal fistula repair are well suited for robotics given the necessity of intracorporeal suturing.

\section{Role of Robotic Surgery in Gynecologic ONCOLOGY}

The role of DRS becomes highly significant in oncology, as the tumor clearance gets translated into survival benefits. Total hysterectomy and staging for endometrial cancer and radical hysterectomy or trachelectomy and pelvic lymphadenectomy for cervical cancer are surgeries where robotic surgery is advised.

\section{Endometrial Cancer and Robotic Surgery}

The standard treatment in most centers is hysterectomy and bilateral salphingo-oopherectomy with pelvic and aortic lymphadenectomy. Tumor grade, depth of invasion, tumor size and lymphovascular space invasion are the main factors deciding the risk of metastasis. The most common limiting factor for comfortable and optimum lymphadenectomy is obesity. The procedure remains a difficult task when approached through laparoscopy. A few reasons cited by the surgeons are: prolonged operating times, fatigue and difficult learning curve. Owing to the better ergonomics and easier learning curve, DRS is fast replacing laparoscopy and open techniques in the treatment of endometrial cancer. Most of the recent studies report a favorable report except for the longer operating time.

Some of the limiting factors noted in laparoscopic surgeries are obesity, narrow pelvis and bulky tumors. Endometrial cancer is commonly associated with obesity and this offers a challenge due to difficult exposure during aortic lymph node dissection and difficulty with ventilating in the steep Trendelenberg position. In such cases, robotics has given better results and is now more preferred over the laparoscopic approach.

\section{Cervical Cancer and Robotic Surgery}

For stage 1A-2 and 1B cervical cancer, radical hysterectomy with pelvic lymphadenectomy is the standard operative treatment. The complication rate, amount of blood loss and mean hospital stay was comparatively lesser. Except for initial studies, other studies show lesser operative time, which may be due to the initial learning curve. For women with undiagnosed cervical cancer who underwent a simple hysterectomy, radical parametrectomy and lymphadenectomy have been suggested as suitable alternatives to pelvic radiation. This procedure has also been tried through robotics at a centerin Houston. Another area of interest is fertility-sparing trachelectomy with pelvic lymphadenectomy. There is not much information available yet on this topic.

In a study by Puntambekar et al. ${ }^{5}$ in Pune, 80 cases were performed robotically. They state that their operative time and estimated blood loss were considerably lower when compared with those of other standard international studies. There was no conversion to open surgery; furthermore no major intraoperative or postoperative complications were noted.

Many other studies, ${ }^{6}$ also suggest that robotic radical hysterectomy (RRH) is preferable over laparoscopic radical hysterectomy (LRH) due to the decrease in blood loss, hospital stay, recovery time, and complications. However, it also depends significantly on the skill of the surgeon.

Larger series-notably among them Boggess et al. ${ }^{7}$ and Lowe et al. ${ }^{8}$ - concluded that there were no transfusions, length of stay in the hospital not more than a day and on the whole the complication rates were significantly lower when compared with open or laparoscopic approaches.

In their experience with 164 oncological surgeries by Puntambekar et al. ${ }^{9} 35$ patients come under the purview of gynecology. They state in their series that the mean operative time was lesser possibly because of extensive open and laparoscopic experience, hybrid techniques, and team effort. They also report that the blood loss was comparatively lesser and they were able to achieve a comparable parametrial, distal vaginal margin and adequate nodal clearance. ${ }^{10}$

In most of the studies, DRS was seen to be associated with lesser blood loss and subsequent blood transfusions with mean hospital stay being significantly lesser. The operative time was comparable between the two groups. More studies need to be done to shed more light on this matter.

\section{Cost Factor}

The main limiting factor hindering the utilization of this technology to its full extent is the cost. The total cost of the surgery can be divided into the following categories:

- Equipment: the DRS (which is being marketed by the Intuitive Surgical Systems, Inc.) is right now the only robotic system available. Owing to the patent that has not yet expired, the market is under monopoly. The capital amount and yearly maintenance costs are hence fixed and exuberant.

- Instrument costs: since most of the working instruments are disposable, they add significantly to the final cost.

- Operation theatre cost: as the working staff, the sterilization techniques and other minor costs remain the same in both, this does not add significantly to the final cost. A question may be raised regarding the possible longer operating hours in robotics 
but that is expected to decrease with the increased experience of the surgeon.

- Length of stay in the hospital: it was seen in most studies to be lesser than the laparoscopic surgeries.

- Other costs: Other expenditure ssuch as consultations with other departments, ICU care, blood transfusions, and so on needs to be accounted for individually.

As seen above it is clear that the major cost is due to the robot itself, the disposable working instruments and its maintenance. Now, on broader inspection we can safely assume that once other Robotic systems come into the market the scenario will change drastically. Though one may argue that the other costs may also contribute significantly, it is seen that robotic surgery is associated with lesser blood loss, lesser complications, and lesser need for intensive care.

Once newer robot systems become available and easily affordable, there may be an overuse of the same even in cases where it is not indicated and offers no advantage over the traditional laparoscopy. The possibility of patients demanding for a robotic surgery should not be neglected. It becomes the responsibility of the robotic surgeons to maintain the rational use of this technology.

\section{Future of Robotic Surgery in India}

Though our country is on the right track with regard to utilization of this technology, much research is needed to make it more productive and cost-effective. This has to be based on critical analysis of evidence-based literature available regarding the same in our institutions. As with any new technology, our outcomes will improve with more experience.

Mass media has a role of paramount importance in creating awareness among the surgeons and patients alike regarding the advantages and accessibility of this technology as there is no dearth of critics. ${ }^{11}$ The government also has to come up with suitable strategies to make this technology accessible to all. Another factor is lack of awareness about the same in many parts of the country due to lack of access to the technology, deficit of learning opportunities and also the innate inability of a few to accept the new technology.

Since the introduction of DRS this revolution has gained momentum and it may just be a matter of years when open and laparoscopic surgeries will be considered outmoded. With the introduction of telesurgery, the prospects are innumerable and not at all beyond imagination.

\section{Conclusion}

India is notably at the brink of a revolution. The need of the hour is to make this new surgically innovative technology accessible to all - to the surgeons as well as the patients. Robotic surgical training is essential for surgeons to help keep up with this revolution so that the man and machine are in sync and move ahead together. It is up to the undoubted talented surgeons of India to embrace this technology to render better quality of medical care to the society.

\section{References}

1. Dogra PN. Current Status of Robotic Surgery in India. JIMSA 2012; 25(3):145-146.

2. Mahesh $D$, Jaspreet $C$, et al. Robotic surgery is ready for prime time in India: for the motion. J Minim Access Surg January-March 2015;11(1):2-4. DOI: 10.4103/0972-9941.147649.

3. Lesley $B C$, Pedro TR, et al. Role of minimally invasive surgery in gynecologic oncology: an updated survey of members of the Society of Gynecologic Oncology. Int J Gynecol Cancer 2015;25(6): 1121-1127. DOI: 10.1097/IGC.0000000000000450.

4. Patricia M, Gilles $\mathrm{H}$, et al. Cost-Effectiveness of Conventional vs Robotic-Assisted Laparoscopy in Gynecologic Oncologic Indications. Int J Gynecol Cancer 2015;25(6):1102-1108. DOI: 10.1097/ IGC.0000000000000458.

5. Shailesh PP, Nallapothula K, et al. Indian Experience of Robotics in Gynecology. J Minim Access Surg 2014;10(2):80-83. DOI: 10.4103/0972-9941.129957.

6. Weinberg $\mathrm{L}$, Rao $\mathrm{S}$, et al. Robotic surgery in gynecology: an updated systematic review. Obstet Gynecol Int 2011;2011(5):852061. DOI: 10.1155/2011/852061.

7. Boggess JF, Gehrig PA, et al. A case-control study of robot-assisted type III radical hysterectomy with pelvic lymph node dissection compared with open radical hysterectomy. Am J Obstet Gynecol 2008;199(357):e1-e7. DOI: 10.1016/j.ajog.2008.06.058.

8. Lowe MP, Chamberlain DH, et al. A multiinstitutional experience with robotic-assisted radical hysterectomy for early stage cervical cancer. Gynecol Oncol 2009;113(2):191-194. DOI: 10.1016/j.ygyno.2009.01.018.

9. Shailesh P, Geetanjali A, et al. Robotic Oncological Survey: Our Initial Experience of 164 Cases. Indian J Surg Onco 2012;3(2):96-100. DOI: 10.1007/s13193-011-0096-0.

10. Hollaway RW, Patel SD, et al. Robotic Surgery in Gynecology. Scan J Surg 2009;98:96-109.

11. Yim GW, Kim SW, et al. Learning curve analysis of robot-assisted radical hysterectomy for cervical cancer: initial experience at a single institution. J Gynecol Oncol 2013;24(4):303-312. DOI: 10.3802/ jgo.2013.24.4.303. 